\title{
Role of Comorbidities as Limiting Factors to the Effect of Hyperbaric Oxygen in Diabetic Foot Patients: A Retrospective Analysis
}

Esra A. Akgül · Jale Karakaya · Salih Aydın

To view enhanced content go to www.diabetestherapy-open.com

Received: August 6, 2014/ Published online: October 2, 2014

(C) The Author(s) 2014. This article is published with open access at Springerlink.com

\section{ABSTRACT}

Introduction: The effectiveness of hyperbaric oxygen therapy (HBOT) on selected diabetic foot wounds continues to be controversial. A holistic approach to diabetes and its comorbidities may be beneficial in the discussion of the proper application of this treatment modality. The aim of the current study is to evaluate the efficacy of HBOT on diabetic foot wounds and provide clinical data that may support this knowledge.

Methods: The present study was a retrospective analysis of the effect of HBOT on diabetic foot lesions ranging 3-5 on the Wagner Grading

Electronic supplementary material The online version of this article (doi:10.1007/s13300-014-0085-8) contains supplementary material, which is available to authorized users.

E. A. Akgül $(\square)$

Fora Hyperbaric Oxygen Therapy Center, Ziyabey C. 1413. S. NO: 4B/5 06520 Balgat, Ankara, Turkey e-mail: akgules@yahoo.com

J. Karakaya

Department of Bioistatistics, Hacettepe University

School of Medicine, Ankara, Turkey

S. Aydin

Department of Underwater and Hyperbaric

Medicine, İstanbul University İstanbul School

of Medicine, Istanbul, Turkey
System. Patients had been treated with HBOT and monitored for 12 months. The results were analyzed in relation to age, gender, diabetes duration and type, microangiopathic complications, peripheral arterial disease (PAD), history of coronary artery disease, stroke, hypertension, smoking habits, glycated hemoglobin, blood sedimentation rate, C-reactive protein, and number of HBOT sessions. Microangiopathies were evaluated as retinopathy, nephropathy, and neuropathy. PAD was determined by available color Doppler ultrasonography and/or angiographic data depending on a modified scoring system. The data of arteries from the aorta to the dorsal pedal artery were scored singly. Average scores of aorto-iliac, femoral, popliteal and pedal levels were also evaluated with this system to compare the healing results in relation to PAD.

Results: One hundred and seventeen patients with 126 diabetic foot wounds were treated. Histories of coronary artery disease, stroke, and non-proliferative or proliferative retinopathy had negative effects on HBOT $(P=0.002$, $P=0.015, P=0.022$, respectively). Depending on the scorings of PAD, the single arterial scores and average scores of aorto-iliac, popliteal and 
pedal levels had no relation to outcomes, while the average scores of the femoral arterial level affected the results $(P=0.048)$.

Conclusions: Diabetic foot patients with histories of coronary artery diseases or stroke and non-proliferative or proliferative retinopathy might resist $\mathrm{HBOT}$. PAD at the femoral arterial level has been shown to have a significant negative effect on HBOT outcomes that should be first considered for surgery. In contrast, PAD below the knee does not seem to be an obstacle to the efficacy of HBOT.

Keywords: Comorbidities; Diabetic foot; Hyperbaric oxygen; Macrovascular complications; Microvascular complications; Peripheral arterial disease; Wound healing; Retinopathy

\section{INTRODUCTION}

Diabetic foot is a worldwide problem that causes morbidity and mortality together with high costs. Nearly $20 \%$ of ulcers are reported as non-healing despite standard care $[1,2]$.

Hyperbaric oxygen therapy (HBOT) is a systemic method to provide extra oxygen to tissues. This therapy achieves sufficient oxygen concentrations for infected hypoxic tissues, and can therefore be added to the treatment of problematic wounds, such as diabetic foot [3]. HBOT enhances neutrophil killing ability and the effect of antibiotics, fibroblast activity, collagen synthesis, and stimulates angiogenesis [4]. Although this treatment is recommended with moderate-quality evidence $[5,6]$, the effectiveness of HBOT on selected diabetic foot wounds still encounters controversy partly because of a history of unsubstantiated claims of its effectiveness in treating a variety of ailments [7]. The complexities of diabetes itself and also of the wounds make prospective, double blind, randomized-controlled trials difficult [1]. There are a few studies including prospective controlled trials on the use of HBOT in diabetic foot [8-13]. The conclusions might be confusing depending on the characteristics of the wounds or the patients with different weighted complications.

The authors of the current study planned to retrospectively evaluate the effect of HBOT on the diabetic foot problem on a wide range of complicated patients. The aim was to discover the role of comorbidities as limiting factors on the effect of hyperbaric oxygen in cases of diabetic foot and to access the clinical data that may support that knowledge.

\section{METHODS}

Patients with diabetic foot wounds were referred to a private, single hyperbaric center and treated with HBOT, mostly as outpatients. Patients were treated conventionally including glycemic control, antibiotherapy, debridement of nonviable tissue and proper wound care at least 2 weeks prior to being referred for HBOT. The reason for referral was no response or worsening of symptoms despite the above measures, or minor amputations in ischemic/ neuroischemic diabetic foot. Patients in this group were not referred if they had just undergone vascular intervention. For neuropathic diabetic foot ulcers, the same treatments were applied at least 1 month prior to the start of HBOT. Patients who had just undergone minor amputations in this group were not accepted for HBOT. Patients who had diabetes for less than 2 years or who also had thromboangiitis obliterans or expansive peripheral arterial disease with early diabetes were also excluded from HBOT. 
Diabetic foot wounds were classified using the Wagner Grading System [14] prior to HBOT. Osteomyelitis was identified by plain radiographs, magnetic resonance imaging or scintigraphy. If the bone was exposed or palpable with a probe, and had purulent leakage, it was accepted to have osteomyelitis.

The demographic data of the patients, diabetes type and duration, smoking habits, history of coronary artery disease, stroke or hypertension, blood sedimentation rates, C-reactive protein (CRP), and glycated hemoglobin (HbA1c) levels at the beginning of HBOT were recorded.

Hyperbaric oxygen therapy sessions were applied in a multiplace chamber (Haux Starmed 2200/5,5; Haux Life Support GmbH, Karlsbad-Ittersbach, Germany) daily, 6 days a week, for a total of $\geq 20$ sessions. All patients were treated with the same HBOT treatment table, consisting of three 25-min oxygen periods, separated by two 5-min air breaks, for a total of $120 \mathrm{~min} / \mathrm{session}$, at 2.4 atmosphere absolute (ATA). A constant medical staff was in charge of the patients' medical management and supervision.

Wound healing was observed at the end of HBOT and 12 months following therapy. Mean follow-up time was $25.6 \pm 13.3$ months (minimum 6; maximum 52). Outcomes were determined by wound healing results. Complete wound closure and no leakage were considered healing. Healing and minor amputations were accepted as favorable. On the other hand, no improvement or major amputations were accepted as unfavorable. Outcomes were analyzed at the end of HBOT depending on the available macrovascular and microvascular data. These outcomes were also compared with the 12th month results.

Peripheral arterial disease (PAD), which was determined by color Doppler ultrasonography
(DUS) and/or by angiographies, was analyzed. A modified scoring system was created to provide a numerical analysis of these tests, depending on previously reported assessment methods [15-17]. The schemes determining the levels and occlusion degrees within the recommended standards for reports dealing with lower extremity ischemia were utilized to assess the angiographic data of the current study [15]. DUS data were scored depending on the arterial flow characteristics. Furthermore, angiographic scoring degrees were inverted to synchronize the values obtained from color DUS data as follows.

Patients who had triphasic waveforms or normal calibration in all arteries from the aorta to the dorsal pedal artery were accepted to be free of PAD, while others were considered to have PAD. Triphasic waveforms on color DUS and/or normal calibration in angiography were scored as 3 points. Biphasic waveform and/or stenosis greater than $50 \%$ was scored as 2 points; monophasic waveform and/or circulation by collateral arteries was scored as 1 point. If there was a total occlusion, it was scored as 0 points. Scores were recorded for each artery from the aorta to dorsal the pedal artery. The average scores of these data were also calculated in four levels as follows:

1. Aorto-iliac (abdominal aorta, common, and external iliac arterial scores).

2. Femoral (common, superficial, and deep femoral arterial scores).

3. Popliteal (popliteal, anterior-posterior tibial arterial scores).

4. Pedal (dorsal pedal artery and plantar arc scores).

The relationship of PAD to wound healing of HBOT-treated patients was evaluated depending on these scores. These patients were also evaluated on their vascular interventions which were decided by surgeons that the patients consulted. 
Patients' microangiopathic and atherosclerotic characteristics were recorded depending on their data given. Microangiopathies were evaluated as retinopathy, nephropathy, and neuropathy. Retinopathy was analyzed in two groups: background retinopathy in one group, and non-proliferated diabetic retinopathy (NPDR) and proliferated diabetic retinopathy (PDR) retinopathy in another group (advanced retinopathy). Nephropathy was analyzed in three groups: normoalbuminuria in one group; microalbuminuria $(30-300 \mathrm{mg} / 24 \mathrm{~h})$ in the second; macroalbuminuria (>300 $\mathrm{mg} / 24 \mathrm{~h}$ ) and end-stage renal disease in the third group. Neuropathy was determined positive if there was a pressure sore or Charcot foot or if the wound occurred due to insensate burn; or if vibratory sensation in the feet was lost. The monofilament test was not used. All microangiopathic data were also studied separately regarding their relations to the outcomes.

The only reported adverse reactions were barotraumatic otitis in 12 patients and a hypoglycemic event in one patient.

\section{Statistical Analysis}

For continuous variables, an independent samples $t$ test was performed to compare the means of two groups. A Mann-Whitney $U$ test was used to compare the two groups for nonparametric data (PAD scorings). A marginal homogeneity test was used to determine the difference of categorical variables between measurements (outcomes at the end of HBOT and at the 12-month follow-up). Pearson's Chi square test, Yates' Chi square test, or Fisher exact tests were used to examine the difference between groups for categorical variables. A logistic regression analysis was performed to analyze the association between the dependent variable (outcomes at the end of HBOT) and independent variables (demographic data, diabetes type and duration, active smoking, history of coronary artery disease, stroke or hypertension, neuropathy, end-stage renal disease and non-proliferative/proliferative retinopathy). The stepwise method was used in the logistic regression analysis. Using the logistic models, odds ratios (OR) and their respective 95\% confidence intervals (CIs) were calculated. A value of $P<0.05$ was accepted as statistically significant. Data analysis was performed using the SPSS 15.0 (Statistical Package for Social Sciences, SPSS Inc. Chicago, IL, USA) software package.

All procedures followed were in accordance with the ethical standards of the responsible committee on human experimentation (institutional and national) and with the Helsinki Declaration of 1975 , as revised in 2000 and 2008. Informed consent was obtained from all patients for being included in the study.

\section{RESULTS}

One hundred and seventeen patients were reviewed, nine of which were readmitted to the hyperbaric center at least 1 year later because of a subsequent wound and these patients were counted twice. Therefore, 126 diabetic foot lesions were evaluated. There were 84 Wagner grade 3, 41 grade 4, and one grade 5 diabetic foot lesions assessed. Of these wounds, $68(54.0 \%)$ were ischemic, six (4.8\%) were neuroischemic, 40 (31.7\%) were neuropathic non-ischemic, and 12 (9.5\%) were neither ischemic nor neuropathic.

The mean age of patients was $59.3 \pm 11.4$ (84 male, 42 female), mean duration of diabetes was $16.8 \pm 8.1$ years, eight patients had type 1 
diabetes and the remaining had type 2 diabetes. Outcomes of HBOT were not related to any of these parameters (Table 1 ).

There were $54(42.8 \%)$ of patients with a history of coronary artery disease and 20 (15.9\%) patients with a history of stroke. These characteristics demonstrated statistically significant relationships to the outcomes of HBOT $(P=0.002$, OR 3.876 and $P=0.015$, OR 4.857 , respectively). Patients with a history of coronary artery disease had a 3.88-fold risk of non-healing or major amputation (95\% CI 1.64-9.16); patients with a history of stroke had a 4.86-fold risk of non-healing or major amputation (95\% CI 1.37-17.28) compared to the other patients with no history. Logistic regression analysis did not reveal any relationship between other comorbidities such as hypertension, active smoking, and presence of neuropathy, advanced retinopathy, or endstage nephropathy and HBOT outcomes (Table 1).

Cigarette pack/years (38.2 \pm 33.6$), \mathrm{HbA} 1 \mathrm{c}$ $(8.9 \pm 1.9 \%)$, blood sedimentation rates $(83.3 \pm 37 \mathrm{~mm} / \mathrm{h})$, CRP levels $(60.7 \pm 62.9)$ and number of HBOT sessions $(47 \pm 23.9)$ had no statistically significant effects on wound healing results (Table 1 ).

Hyperbaric oxygen therapy was applied for $47 \pm 23.9$ (minimum: 20, median: 42) sessions. At the end of HBOT, 39.6\% wounds were healed completely, $20.6 \%$ received minor amputation, $27.0 \%$ showed no improvement and $12.7 \%$ received major amputation. There were no statistically significant differences of the wound healing results compared with the 12-month follow-up results excluding patients who passed away within this period $(P=0.214$, Table 2).

Peripheral arterial disease was determined by angiographic and/or color Doppler ultrasonographic data in 86 patients. Twenty-
Table 1 Patient characteristics and relations to HBOT outcomes

\begin{tabular}{|c|c|c|}
\hline Patient characteristics & $\begin{array}{l}\text { Patients } \\
n \text { (total } \\
n=126)\end{array}$ & $\begin{array}{l}\text { Relation to } \\
\text { outcomes } \\
\text { at the end } \\
\text { of HBOT } \\
\text { (P values) }^{\text {a }}\end{array}$ \\
\hline \multicolumn{3}{|l|}{ Logistic regression $^{\mathrm{b}}$} \\
\hline Mean age $\pm S D$ & $59.3 \pm 11.4$ & n.s. \\
\hline Sex $M / F$ & $84 / 42$ & n.s. \\
\hline $\begin{array}{l}\text { Mean diabetes duration } \\
\text { (years) } \pm \mathrm{SD}\end{array}$ & $16.8 \pm 8.1$ & n.s. \\
\hline Type 1 type 2 DM & $8 / 118$ & n.s. \\
\hline Neuropathy $+/$ total & $103 / 126$ & n.s. \\
\hline ESRD/total & $33 / 126$ & n.s. \\
\hline NPDR and PDR/total & $91 / 107$ & n.s. \\
\hline $\begin{array}{l}\text { History of coronary artery } \\
\text { dis. }\end{array}$ & $54(42.9 \%)$ & 0.002 \\
\hline History of stroke & $20(15.9 \%)$ & 0.015 \\
\hline Hypertension & $73(57.9 \%)$ & n.s. \\
\hline Active smokers & $67(53.2 \%)$ & n.s. \\
\hline \multicolumn{3}{|l|}{ Independent sample $t$ test $^{\mathrm{c}}$} \\
\hline $\begin{array}{l}\text { Mean cigarette } \\
\text { pack/year } \pm \text { SD }\end{array}$ & $38.2 \pm 33.6$ & n.s. \\
\hline Mean HbAlc $(\%) \pm S D$ & $8.9 \pm 1.9$ & n.s. \\
\hline $\begin{array}{l}\text { Mean sedimentation } \\
\qquad(\mathrm{mm} / \mathrm{h}) \pm \mathrm{SD}\end{array}$ & $83.3 \pm 37$ & n.s. \\
\hline Mean CRP $(\mathrm{mg} / \mathrm{dl}) \pm \mathrm{SD}$ & $60.7 \pm 62.9$ & n.s. \\
\hline $\begin{array}{l}\text { Mean number of } \mathrm{HBO} \\
\text { sessions } \pm \mathrm{SD}\end{array}$ & $47 \pm 23.9$ & n.s. \\
\hline
\end{tabular}

CRP C-reactive protein, $D M$ diabetes mellitus, ESRD End-stage renal disease, $H b A 1 c$ glycated hemoglobin, $H B O T$ hyperbaric oxygen therapy, NPDR nonproliferative diabetic retinopathy, n.s. non-significant, PDR proliferative diabetic retinopathy

a Outcomes were consisted of healed or minor amputated patients in the favorable group; non-healed or major amputations in the unfavorable group

Statistical analysis method used was: ${ }^{b}$ logistic regression, ${ }^{c}$ independent sample $t$ test 
Table 2 Outcomes of HBOT

\begin{tabular}{|c|c|c|c|}
\hline Status of patient & At completion of HBOT $n, \%^{\mathrm{a}}$ & At 12th month $n, \%^{\mathrm{a}}$ & At $25.6 \pm 13.3$ months $n, \%$ \\
\hline Healed & $50(39.7 \%)$ & $55(49.1 \%)$ & $46(47.9 \%)$ \\
\hline Minor amputation & $26(20.6 \%)$ & $21(18.8 \%)$ & $20(20.8 \%)$ \\
\hline No improvement & $34(27.0 \%)$ & $21(18.8 \%)$ & $15(15.6 \%)$ \\
\hline Major amputation & $16(12.7 \%)$ & $15(13.4 \%)$ & $15(15.6 \%)$ \\
\hline Total wound & 126 & 112 & 96 \\
\hline Deceased & 0 & 14 & 30 \\
\hline Total patient & 117 & 103 & 87 \\
\hline
\end{tabular}

$n$ number, HBOT hyperbaric oxygen therapy

a There were no statistically significant differences for wound healing results at completion of HBOT and at the 12th month $(P=0.214)$

seven of these patients had normal arterial conditions while the remaining had PAD. Most of the patients with PAD were not operated on because a lack of optimal vascular interventions. Among these 51 non-operated patients, 21 (41.2\%) had atherosclerotic changes placed below the knee, 30 (58.8\%) had these changes placed both above and below the knee (Fig. 1).

Depending on the modified scoring system, no significant relationship between the outcomes and the arterial scorings was found when each artery was evaluated singly. However, when considered by average scorings of four levels (aorto-iliac, femoral, popliteal, and pedal), only the average of the femoral arterial scores significantly affected the results $(P=0.048$, Table 3$)$. The mean value of femoral arterial scores was $2.77 \pm 0.5$ (median 3 , min 1 , $\max 3$ ) in the first (favorable) group and $2.64 \pm 0.4$ (median 2.67, $\min 1, \max 3$ ) in the second (unfavorable) group.

The effect of microangiopathic complications on the outcomes was also studied separately. Advanced retinopathy significantly affected the outcomes $(P=0.022$,

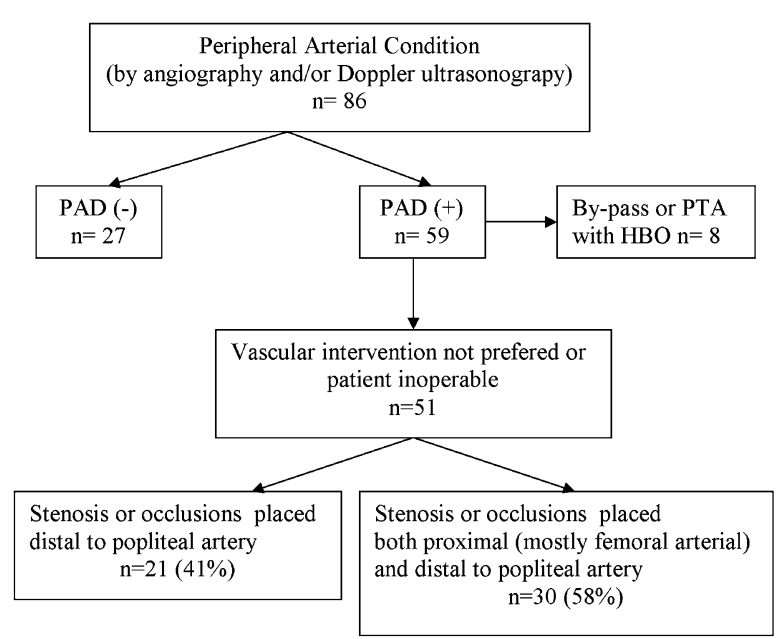

Fig. 1 Peripheral arterial condition of patients who have angiographic and/or color DUS data. PAD was determined by color DUS and/or by angiographies. DUS Doppler ultrasonography data, $H B O$ hyperbaric oxygen, $P A D$ peripheral arterial disease, $P T A$ percutaneous transluminal angioplasty

Table 4). However, when nephropathy was analyzed in three groups (normoalbuminuria, microalbuminuria, and later stages) or in two groups (end-stage renal disease and others), it had no relation to the outcomes $(P=0.423$ and $P=0.060$, respectively). Similarly, neuropathy $(P=0.706)$ had no relation to the outcomes. 
Table 3 Relation of PAD to HBOT outcomes

\begin{tabular}{ll}
\hline $\begin{array}{l}\text { Scoring levels of the } \\
\text { arterial tests }\end{array}$ & $\begin{array}{l}\text { Relation of scores } \\
\text { to outcomes } \\
(\boldsymbol{P} \text { values })^{\mathbf{b}}\end{array}$ \\
\hline Aorto-iliac level (average) & 0.963 \\
Abdominal aorta & 1.000 \\
Common internal artery & 0.707 \\
External iliac artery & 0.531 \\
Femoral level (average) & 0.048 \\
Common femoral artery & 0.185 \\
Deep femoral artery & 0.101 \\
Superficial femoral artery & 0.070 \\
Popliteal level (average) & 0.215 \\
Popliteal artery & 0.277 \\
Anterior tibial artery & 0.996 \\
Peroneal artery & 0.398 \\
Posterior tibial artery & 0.069 \\
Pedal level (average) & 0.316 \\
Dorsal pedal artery & 0.347 \\
\hline Plantar arc & 0.662 \\
\hline
\end{tabular}

Relations of PAD to outcomes were analyzed depending on Doppler US and/or angiographic data of 86 patients at the end of HBOT

DUS Doppler ultrasonography, HBOT hyperbaric oxygen therapy, $P A D$ peripheral arterial disease

a These arterial tests were worked up as explained in the text and scores at the levels shown in the table were compared with the outcomes

b Outcomes were consisted of healed or minor amputated patients in the favorable group; non-healed or major amputated patients in the unfavorable group

\section{DISCUSSION}

There are a few prospective controlled trials about HBOT usage on diabetic foot. Although conclusions are different in these studies, each of them has been focused on different types of wounds and patients [8-12]. The authors of the current study retrospectively evaluated the effect of HBOT on diabetic foot wounds in a wide range of patients with varying complications. The aim was to discover the factors that affect the outcome of HBOT and to understand the factors determining its efficacy.

Peripheral arterial disease is a known risk factor for limb amputation in patients with diabetes [18-20]. Angiographic and Doppler US scoring are reported as assessment methods to estimate the degree of PAD [15-17]. In the present study, the researchers modified these methods and evaluated the data of color DUSs and/or angiographies of 86 patients. Although ankle: brachial pressure scorings were shown to be in close correlation with arteriographic scorings [16], the researchers preferred to use the flow characteristics of the arterial DUS data because of the unreliability of the Dopplerderived ankle: brachial index depending on arterial media calcification of diabetic vascular disease [21]. Furthermore, angiographic scoring degrees were inverted to synchronize the values obtained from color DUS. These modifications allowed the assessments to be made together or

Table 4 Relation of retinopathy to outcomes

\begin{tabular}{|c|c|c|c|c|c|}
\hline & Healed $n, \%$ & $\begin{array}{l}\text { Minor } \\
\text { amputated } n, \%\end{array}$ & $\begin{array}{l}\text { Non-healed } \\
n, \%\end{array}$ & $\begin{array}{l}\text { Major } \\
\text { amputated } n, \%\end{array}$ & Total $n$ \\
\hline Background retinopathy & $11(69 \%)$ & $0(0 \%)$ & $4(25 \%)$ & $1(6 \%)$ & $16(100 \%)$ \\
\hline NPDR/PDR & $30(33 \%)$ & $23(25 \%)$ & $26(29 \%)$ & $12(13 \%)$ & $91(100 \%)$ \\
\hline Total patients & 41 & 23 & 30 & 13 & 107 \\
\hline
\end{tabular}

There was a significant relation of outcomes to retinopathy at the end of HBOT $(P=0.022)$

$H B O T$ hyperbaric oxygen therapy, NPDR non-proliferative diabetic retinopathy, $P D R$ proliferative diabetic retinopathy 
separately by the two different methods providing anatomic and hemodynamic information.

Among the 51 non-operated PAD patients of the current study, 21 (41\%) had atherosclerotic changes placed below the knee, 30 (58\%) had these changes placed both above and below the knee (Fig. 1) in accordance to atherosclerosis of diabetes [22, 23].

The present study used the arterial scorings of these patients to compare the PAD conditions and outcomes of those who were treated with HBOT. The scorings of each artery from the aorta to the pedal arteries were not associated with the outcomes. Although not significant, the $P$ values related to the superficial femoral and posterior tibial arterial scorings ( $P=0.070, \quad P=0.069$, respectively) were distinctly lower than other single arteries (Table 3). On the other hand, depending on the average scorings of the four arterial levels, the results demonstrate that there is a significant relationship to HBOT outcomes at the femoral arterial level $(P=0.048$, Table 3$)$, but not at the others. This suggests that wherever atherosclerotic changes were placed, only the femoral arterial locations affect the outcomes of HBOT negatively. Therefore, in the presence of femoral atherosclerotic lesions, attention must be given to vascular interventions before HBOT is initiated. In contrast, PAD below the knee does not seem to be an obstacle to the efficacy of HBOT in the treatment of diabetic foot.

PAD is an expression of systemic atherosclerotic disease. Therefore, the researchers of the current study evaluated the patients' histories of coronary artery disease, hypertension, stroke, and smoking habits to assess the other data related to atherosclerosis. A history of coronary artery disease and stroke has been demonstrated to worsen wound healing of the diabetic foot patients treated with HBOT ( $P=0.002$ and $P=0.015$, respectively). This is true about coronary artery disease, regardless of HBOT [1, 24]. Faglia et al. [25] demonstrated prior instances of stroke to be a prognostic determinant of major amputation in patients treated with or without HBOT. In conclusion, one should take coronary artery disease and stroke history into consideration because of their possible negative effect on expected outcome.

Microvascular complications are other comorbidities that affect wound healing of patients with diabetes. The current study evaluated the relationships of HBOT outcomes to neuropathy, nephropathy, and retinopathy. Although neuropathy was not shown to affect the outcomes significantly, the quality of this argument is poor. A strong association with both diabetic foot ulceration and lower extremity amputation has been demonstrated with chronic kidney disease and end-stage renal disease [26, 27]. In the current study, interestingly there was not a significant relationship to chronic kidney disease or endstage renal disease. Despite a small amount of missing data on retinopathy, the researchers have demonstrated that HBOT outcomes were significantly better when retinopathy was at a background stage and poorer when it progressed $(P=0.022$, Table 4$)$. One might hypothesize that the progression of retinopathy might be an early and more valuable marker when discussing about the effect of HBOT on wound healing. These data should be taken to consideration in further trials as they can provide clues about the mechanism of the effect of HBOT on microcirculation which may facilitate wound healing.

A long-term analysis of the effect of HBOT on ischemic diabetic ulcers has shown that the benefit of HBOT seemed to persist after 
discontinuing the therapy $[9,10]$. These results may greatly depend on the effect of angiogenesis [8]. In accordance with this, there were no statistically significant differences of the favorable outcomes compared with the 12-month follow-up results excluding patients who passed away within this period (Table 2).

Patients' characteristics such as age, diabetes duration and HbA1c were not shown to be related to the outcomes, contrary to expectations. This might be due to the complexity of the other factors such as comorbidities and the extent of PAD which were shown to primarily affect healing [1].

A lack of a reliable assessment of the neuropathy was a limitation of this study. Diabetic foot off-loading techniques were applied very poorly both before and with HBOT. Retinopathy could have not been evaluated in all patients. Transcutaneous oxygen pressure (TcPO2) measurements which may have been valuable were not made.

Diabetic foot should be considered a sign of multi-organ disease. Therefore, before a decision about HBOT has been reached, an overall understanding of the patients' condition of diabetes, as well as the wound, is required. The current study demonstrates that histories of coronary artery disease or stroke, and advanced retinopathy worsen the outcomes of HBOT significantly. The results also revealed that PAD at the femoral level should be first considered for surgery, but PAD below the knee seems not to be an obstacle to the effect of HBOT in the treatment of diabetic foot.

\section{ACKNOWLEDGMENTS}

No funding or sponsorship was received for this study or publication of this article. All named authors meet the ICMJE criteria for authorship for this manuscript, take responsibility for the integrity of the work as a whole, and have given final approval to the version to be published.

Conflict of interest. Esra A Akgül, Jale Karakaya, Salih Aydin declare no conflict of interest.

Compliance with ethics guidelines. All procedures followed were in accordance with the ethical standards of the responsible committee on human experimentation (institutional and national) and with the Helsinki Declaration of 1975, as revised in 2000 and 2008. Informed consent was obtained from all patients for being included in the study.

Open Access. This article is distributed under the terms of the Creative Commons Attribution Noncommercial License which permits any noncommercial use, distribution, and reproduction in any medium, provided the original author(s) and the source are credited.

\section{REFERENCES}

1. Gershater MA, Löndahl M, Nyberg P. Complexity of factors related to outcome of neuropathic and neuroishaemic/ischaemic diabetic foot ulcers: a cohort study. Diabetologia. 2009;52(3):398-407.

2. Oyibo SO, Jude EB, Tarawneh I, et al. The effects of ulcer size and site, patient's age, sex and type and duration of diabetes on the outcome of diabetic foot ulcers. Diabet Med. 2001;18(2):133-8.

3. Roeckl-Weidmann I, Bennett $M$, Kranke P. Systematic review of hyperbaric oxygen in the management of chronic wounds. $\mathrm{Br} \mathrm{J}$ Surg. 2005;92:24-32.

4. Warriner RA III, Hopf HW. Enhancement of healing in selected problem wounds. In: Feldmeier JJ, editor. Hyperbaric oxygen 2003: indications and 
results. The hyperbaric oxygen therapy committee Report. Maryland: UHMS; 2003. p. 41-55.

5. Lipsky BA, Berendt AR, Cornia PB, et al. Infectious diseases society of america clinical practice guideline for the diagnosis and treatment of diabetic foot infections. Clin Infect Dis. 2012;54(12):1679-84.

6. Steed DL, Attinger C, Colaizzi T. Guidelines for the treatment of diabetic ulcers. Wound Repair Regen. 2006;14(6):680-92.

7. Bishop AJ, Mudge E. Diabetic foot ulcers treated with hyperbaric oxygen therapy: a review of the literature. Int Wound J. 2014;11(1):28-34.

8. Faglia E, Favales F, Aldeghi A, et al. Adjunctive systemic hyperbaric oxygen therapy in treatment of severe prevalently ischemic diabetic foot ulcer. Diabetes Care. 1996;19(12):1338-43.

9. Abidia A, Laden G, Kuhan G, et al. The role of hyperbaric oxygen therapy in ishaemic diabetic lower extremity ulcers: a double-blind randomizedcontrolled trial. Eur J Vasc Endovasc Surg. 2003;25(6):513-8.

10. Kalani M, Jörneskog G, Naderi N, et al. Hyperbaric oxygen (HBO) therapy in treatment of diabetic foot ulcers long-term follow-up. J Diabetes Complicat. 2002;16(2):153-8.

11. Kessler L, Bilbault P, Ortéga F, et al. Hyperbaric oxygenation accelerates the healing rate of nonischemic chronic diabetic foot ulcers. Diabetes Care. 2003;26(8):2378-82.

12. Löndahl M, Katzman P, Nilsson A, Hammarlund C. Hyperbaric oxygen therapy facilitates healing of chronic foot ulcers in patients with diabetes. Diabetes Care. 2010;33(5):998-1003.

13. Margolis DJ, Gupta J, Hoffstad O, et al. Lack of effectiveness of hyperbaric oxygen therapy for the treatment of diabetic foot ulcer and the prevention of amputation. Diabetes Care. 2013;36(7):1961-6.

14. Armstrong DG, Lavery LA, Harkless LB. Validation of a diabetic wound classification system. The contribution of depth, infection, and ischemia to risk of amputation. Diabetes Care. 1998;21:855-9.

15. Rutherford RB, Baker JD, Ernst C, et al. Recommended standards for reports dealing with lower extremity ischemia: revised version. J Vasc Surg. 1997;26:517-38.

16. Shearman CP, Gwynn BR, Curran F, et al. Noninvasive femoropopliteal assessment: is that angiogram really necessary? Br Med J (Clin Res Ed). 1986;293(6554):1086-9.
17. Mazzariol F, Ascher E, Hingorani A. Lower extremity revascularization without preoperative contrast arteriography in 185 cases: lessons learned with duplex ultrasound arterial mapping. Eur J Vasc Endovasc Surg. 2000;19:509-15.

18. Miyajima S, Shirai A, Yamamoto S, et al. Risk factors for major limb amputations in diabetic foot gangrene patients. Diabetes Res Clin Pract. 2006;71(3):272-9.

19. Ince $\mathrm{P}$, Kendrick D, Game F, Jeffcoate W. The association between baseline characteristics and the outcome of foot lesions in a UK population with diabetes. Diabet Med. 2007;24(9):977-81.

20. Brechow A, Slesaczeck T, Münch D, et al. Improving major amputation rates in the multicomplex diabetic foot patient: focus on the severity of peripheral arterial disease. Ther Adv Endocrinol Metab. 2013;4(3):83-94.

21. Emanuele MA, Bauchanan BJ, Abraira C. Elevated leg systolic pressure and arterial calcifications in diabetic vascular occlusive disease. Diabetes Care. 1981;4(2):289-92.

22. Faglia E. Characteristics of peripheral arterial disease and its relevance to the diabetic population. Int $\mathrm{J}$ Low Extrem Wounds. 2011;10(3):152-66.

23. Conte MS. Diabetic revascularization: endovascular versus open bypass-do we have the answer? Semin Vasc Surg. 2012;25(2):108-14.

24. Adler AI, Boyko EJ, Ahroni JH, Smith DG. Lowerextremity amputation in diabetes. The independent effects of peripheral vascular disease, sensory neuropathy, and foot ulcers. Diabetes Care. 1999;22(7):1029-35.

25. Faglia E, Favales F, Aldeghi A, et al. Change in major amputation rate in a center dedicated to diabetic foot care during the 1980s: prognostic determinants for major amputation. J Diabetes Complicat. 1998;12(2):96-102.

26. Ndip A, Rutter MK, Vileikyte L, et al. Dialysis treatment is an independent risk factor for foot ulceration in patients with diabetes and stage 4 or 5 chronic kidney disease. Diabetes Care. 2010;33(8):1811-6.

27. Margolis DJ, Hofstad O, Feldman HI. Association between renal failure and foot ulcer or lowerextremity amputation in patients with diabetes. Diabetes Care. 2008;31(7):1331-6. 